\title{
On Realization of Self-Adaptive Personalized Knowledge Service Based on Context Aware
}

\author{
Yongchang Jiang \\ Library. Harbin University Of Commerce \\ Harbin University Of Commerce, HUC \\ Harbin, China \\ E-mail: jiangych@hrbcu.edu.cn
}

\author{
Jing Zhang \\ Library, Northeast Forestry University \\ Northeast Forestry University, NFU \\ Harbin, China \\ E-mail: xxfw7@163.com
}

\begin{abstract}
In Web2.0, users become the adaptive agents of knowledge architecture. As a result, knowledge architecture and services get restored to their original state of human-oriented and furthermore, personal knowledge architecture becomes a process of self-organization. This provides a favorable condition under which we can expect to develop a self-adaptive personalized knowledge service system based on context-aware. However, the implementation of such a system will not be possible without utilizing the standard semantics descriptions and inferences from ontology when processing knowledge items and context information.
\end{abstract}

Keywords-Web2.0; Personalized Knowledge service; Context-aware; Ontology

\section{INTRODUCTION}

As an open complex adaptive knowledge system, Web2.0 uses some applications like Blog, Wiki, Tag, SNS, RSS as its kernel, and puts many complex new theories and technologies, for example, Six degrees separation, Synergetics, Long tail, XML, Ajax into the background. So that knowledge production, communication and utilization have realized in self-organization and ordering through users going into them widely under the restriction of simple rulers by the patterns of multidimension, diversification, personalization and decentralization[1]. This above not only enables users to become the adaptive agents of knowledge architecture in Web2.0, revert knowledge architecture to its nature state of human- oriented and gives users rich experiences; but also enables personal knowledge architecture to reflect the self- organizing principle[2]. As a result, we can expect the realization for personalized knowledge service (PKS) which is based on context-aware.

Context-aware in PKS means providing a user with knowledge and service that suit the user's current situation (such as the user' goals and tasks) through automatically discovering and obtaining the user' needs based on the awareness of the context information of user. The goals for context-aware in PKS are to realize a system self-adaptation between user needs and the knowledge service provided, to improve the accuracy and reliability of PKS, and to help improving the quality and efficiency of knowledge service system [3,4]. However, the realization of self-adaptation in PKS requires utilizing the standard semantics descriptions and inferences from ontology in order for the PKS system to be able to discover users' current and historical contexts, based on which the knowledge presented to users can be automatically adjusted. As a consequence, the system can expect to meet a user's specific and personalized needs for knowledge.

\section{THE ADVANTAGES OF CONTEXT-AWARE PKS IN Web 2.0}

In the open complex adaptive Web 2.0 knowledge system, every adaptive agent can perform its personal knowledge architecture by self-organization in competi- tion and collaboration with other agents ${ }^{[2]}$. Relying on its capabilities of self-organization and self-adaptation, the agent will change its own knowledge structure and behavior patterns in order to be continuously adaptive in personal knowledge architecture during subsequent loose group integration and non-linear interactions. Meanwhile, the knowledge service system will become more self-adaptive and more customized in terms of user knowledge acquisition, application and creation. Therefore, Web2.0 provides a favorable environment under which an self-adaptive PKS system based on context-aware can be established.

\section{A. Meet users' personal needs for knowledge through automatically obtaining context information}

In the process of knowledge acquisition, a user's personal needs are context-sensitive ${ }^{[5]}$. Only a context-aware knowledge service system can ensure 
the accuracy and reliability in discovering user personal knowledge needs and self-adaptability in matching between user needs and services ${ }^{[6]}$. However, many existing PKS systems are either too hard or too complicated. For example, users have to input either their relevant personal information (such as their interests/hobbies, research areas, etc.) or other feedback before receiving services. But in Web2.0, the system is able to directly or indirectly discover a user's current context information, such as what the user is currently doing and what the user is going to do, based on the most recent tagging a user has tended to, the list of Blog subjects that the user has read, or the disciplinary area that the user has been involved in Wiki. The system will then put this context informa-tion into a collection of universally described contexts in order to obtain the semantics descriptions of the context information through using $\mathrm{OWL}^{[7]}$ ontology. Since an OWL ontology is essentially a series of ontology concepts and the relationships among these concepts, the semantically described user contexts can be represented by a matrix of weighted ontology concepts-uc, uc $\in[0,1]|0|$. The weight represents a correlation between the concept and the context information.

\section{B. Improve the accuracy and reliability of PKS through} context information renew

In a PKS system, a user's behavior usually follows a continuing pattern, so do its requirements for knowledge. A user's current context only partially reflects the user's immediate needs. Historical context should also be considered in order to realize personalization of context-aware knowledge services. In order to accurately discover a user' needs for knowledge an overall context needs to be developed through integrating a user's current context with its historical context. Therefore, such an overall context consists of two parts-historical context and current context. Historical context is an accumulation of historical events from time to time and it can be used to develop subsequent contexts information. A user's current context is discovered through user's immediate actions at the moment. The following is the formula for an overall context:

$$
u d(t)=\zeta \cdot u d(t-1)+(1-\zeta) \cdot u c_{\text {current }}(t)
$$

$\xi$-Forgetting factor;

uc(t-1) - the overall historical contexts up to time $\mathrm{t}$;

$\mathrm{uc}_{\text {current }}(\mathrm{t})$ - the current context at timet.

The forgetting factor $-\xi$ refers to the weighting factor of historical contexts. The more distant the past is, the smaller the forgetting factor $\xi$ becomes, resulting in a weaker historical impact. $\xi \in[0,1], \mathrm{A} \xi$ of 0 means historical context is ignored and only the current context is considered. At the starting point when $\mathrm{t}=0$, the overall context is equal to the current context: $\mathrm{uc}(0)=\mathrm{uc}_{\text {current }}(0)$.

Based on the formula, the forgetting factor can also be adjusted to reflect the impact of the current context on the personalization of knowledge services. It needs to be adjusted down if the impact of the current context is assumed to be stronger, and vice versa. Preliminary attempts have proved that the ideal results can be achieved if the value of $\xi$ is set to be between 0.1 and 0.5 . The process of context dynamic renewing and integrating as is the following illustration:

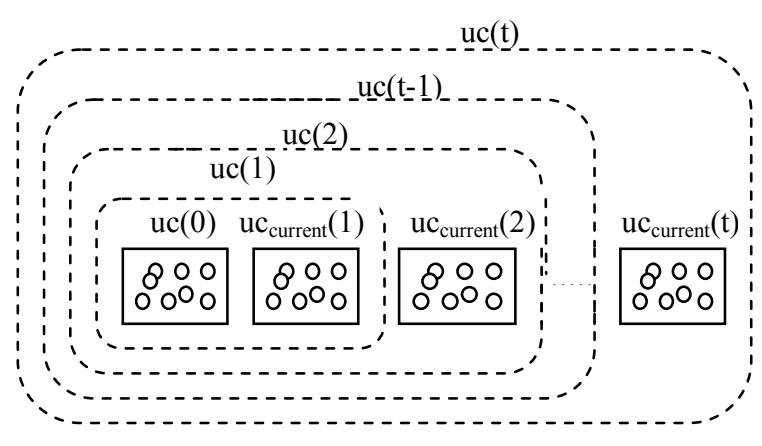

Fig1 context dynamic renewing and integrating

\section{Realize self-adaptability in PKS through personal semantic matching}

In Web 2.0, user requirements for knowledge are usually complex, multi-dimensional, heterogeneous, changeable or even self-conflicting. Therefore, the PKS system should be able to discover the changes in user requirements caused by the changed goals, tasks or circumstances and eventually be able to adjust the knowledge provided to its users based on the these changes.

A personal semantic match is to determine the similarity degree between a knowledge item (d) and an overall context at a time point $\mathrm{t}(\mathrm{uc}(\mathrm{t}))$ by using some matching algorithms. The goal is to improve the self-adaptability of PKS. Since an overall context and a knowledge item that determine the similarity degree $(\mathrm{pms}(\mathrm{d}, \mathrm{uc}(\mathrm{t})))$ are both extended ontology semantics descriptions, the first step in the process is to extend the ontology semantics labels of the knowledge item and the context before calculating the matching degree among ontology semantic concepts. The process of personal semantic similarity matching as is show in figure2: 


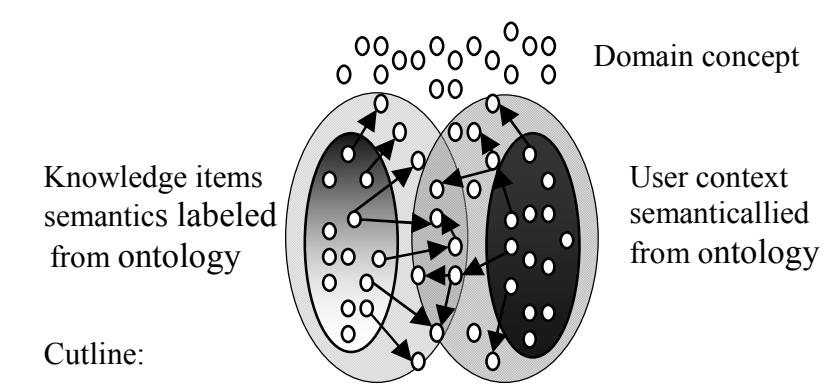

starting ontology semantics labels of knowledge items

--) extended ontology semantics labels of knowledge items

starting ontology semantics descriptions of user contexts

extended ontology semantics descriptions of user contexts

matching semantics ontology concept

Fig2 The process of personal semantic similarity matching based on extended ontology semantics

There are various algorithms used to calculate the match degree between extended semantics concepts of a knowledge item and an overall context. A researcher has proposed an algorithm called similarity evaluation method based on context-aware trees ${ }^{[8]}$. Given that the ontology semantics of a knowledge item and an overall context can both be represented by a matrix of weighted semantics concepts, we can adopt the cosine function similarity degree match algorithm in matrix:

$$
\operatorname{pms}(d, u c(t))=\cos (d, u c(t))=\frac{d \cdot u c(t)}{|d| \cdot|u c(t)|}
$$

\section{Improve PKS through detection}

Users are not always certain about their requirements for knowledge, therefore, a PKS system need to know a user's previous knowledge in order to expand its knowledge collection based on which the system is able to discover the user's potential interests and future needs for knowledge. As a result, the system will be able to meet users' requirements for PKS.

In order for the results returned by a knowledge service system to reflect both a user search and the personal contexts, the system needs to integrate the above personal match similarity degree $(\mathrm{pms}(\mathrm{d}, \mathrm{uc}(\mathrm{t})))$ with the non-personalized match degree between a user search and a knowledge item $(\operatorname{sim}(\mathrm{d}, \mathrm{q}))$ in order to determine a final ranking score $(\operatorname{score}(\mathrm{d}, \mathrm{q}, \mathrm{uc}(\mathrm{t})))$ of a knowledge item. The following is the formula:

$$
\operatorname{scor}(d, q, u c(t))=f(\operatorname{pms}(d, u d(t), \operatorname{sim}(d, q)))
$$

In the above formula, the similarity degree between a knowledge item and a user query can be provided by a traditional knowledge retrieval system. It can also be obtained through ranking the knowledge items in various Web 2.0 applications, such as Tag or Blog. This means $\operatorname{sim}(\mathrm{d}, \mathrm{q})$ does not depend on a specific knowledge retrieval system. Therefore the method can be adopted in an existing knowledge retrieval system.

A core issue is to determine the function $\mathrm{f}$ through which an overall ranking score can be obtained ${ }^{[9]}$. The following is a formula to obtain a ranking score:

$$
\operatorname{score}(d, q, u c(t))=\lambda \cdot \operatorname{pms}(d, u c(t))+(1-\lambda) \cdot \operatorname{sim}(d, q)
$$

$\lambda$ is a variable for personalization, $\lambda \in[0,1]$.The value of $\lambda$ determines the extent to which personal contexts affect the end results provided by a knowledge service system. A $\lambda$ value of 0 means that a user's personal contexts are completely ignored and the service is no different than that provided by a traditional knowledge retrieval system. A $\lambda$ value of 1 means a user's query is ignored and the results from knowledge service depend totally on the user's personal contexts, this could be used in system-initiated knowledge services. A higher $\lambda$ indicates a bigger impact of personal contexts, and vice versa.

\section{CONSTRUCTION AND REALIZATION OF A}

\section{CONTEXT-AWARE SELF-ADAPTIVE PKS SYSTEM}

Based on the advantages of self-adaptive PKS based on context-aware system in Web 2.0 and the possibility of utilizing the standard semantics descriptions and inference from ontology, we could design and implement a self-adaptive PKS system based on context-aware. The following is a framework of such a system:

In the framework, a user consciously interacts with knowledge content and other users on a certain topic area through using various Web2.0 applications. The user is able to carry on certain tasks through self-adaptation. When the user becomes certain about his/her requirements for a specific knowledge item, he/she will submit a keyword search to a corresponding knowledge retrieval system which will return a series of matching results. The order of the results is determined based on the similarity degree $\operatorname{sim}(d, q)$ between the user query (q) and a knowledge item (d). This is no different than traditional knowledge retrieval because a user's personal contexts are ignored. In the context-aware PKS system, the results from traditional knowledge retrieval system are not directly provided to the user. What the knowledge retrieval system does is, first, labeling the knowledge items based on ontology semantics resulting in a series of semantically described knowledge items. In the mean time, the system obtains the user's current context information $\mathrm{uc}_{\text {current }}(\mathrm{t})$ based on user's actions in various Web2.0 applications, messages from relevant work flow engines, recorded user behaviors, and other information from both internal or external sources. Instead of being used directly in PKS, the current context information is integrated with historical context information resulting in an overall user' context uc(t) labeled semantically from ontology. Since the 
knowledge items and the overall contexts are both labeled by utilizing ontology semantics, the similarity degree can be calculated by using an algorithm. The final results from PKS system compares the personal similarity degree pms $(\mathrm{d}, \mathrm{uc}(\mathrm{t}))$ and the non-personalized similarity degree $\operatorname{sim}(d, q)$ between a knowledge item and a user query before it returns a series of results with ranking orders. A user would then be able to choose certain results to carry on its tasks. These actions are automatically recorded in the system and become the source for subsequent developments of historical contexts. As a result, a user's context information keeps being renewed and knowledge service becomes self-adaptive to the constantly changing user contexts.

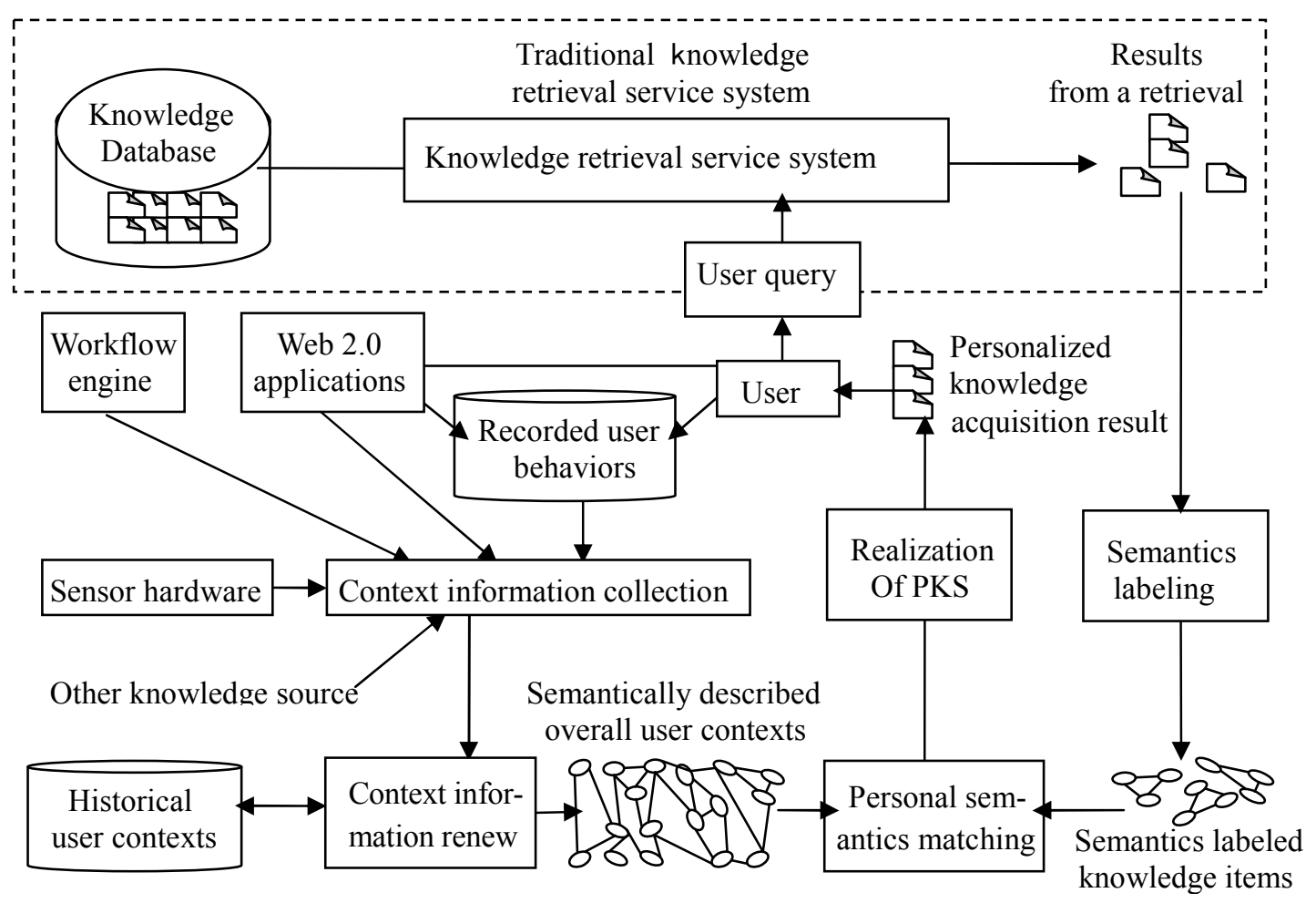

Fig3 Framework of a self-adaptive PKS system based on context-aware

\section{CONCLUSIONS}

The self-organization mechanism of personal knowledge architecture in Web2.0 can be applied to establish a self-adaptive PKS system based on contextaware. The system needs to automatically and constantly obta- in and renew user context information in order to make its services accurate, reliable and selfadaptive. The system also needs to discover user requirements through developing an overall context by utilizing semantics descriptions and inferences from ontology. The system then display the results from these self-adaptive PKS based on the similarity degrees between the overall context and a knowledge item. The ultimate goal is to realize self-adaptive PKS based on context-aware.

\section{REFERENCES}

[1] Feicheng Ma. The mechanism of ordering information in Web $2.0[\mathrm{~J}]$. Document, Information and Knowledge, 2009(6):14-18

[2] Yongchang Jiang. Knowledge architecture based on user experience - A review of the basic principles of KA in Web2.0 [J]. Journal of the China Society for Scientific and
Technical Information, 2010(5):872-879.

[3] Yang S, Shao N. Enhancing pervasive Web accessibility with rule-based adaptation strategy[J]. Expert Systems with Applications, 2007(32): 1154-1167.

[4] Guangyou Xu, Yuanhun Shi, Weikai Xie. Pervasive/ ubiquitous computing $[\mathrm{J}]$. Chinese Journal of Computers, 2003,26(9):1042-1050.

[5] Shuliang Zhang, Fuhai Leng. Acquisition of personalized information and realization of personalized services in the Web Environment[J]. Journal of Library Science In China,2007,33(4): 77-81.

[6] Mylonas P H, Vallet D, et al Personalized information retrieval based on context and ontological knowledge [J]. The Knowledge Engineering Review, 2008, 23 (1): 73-100.

[7] W3C. OWL Web Ontology Language Guide Recommenda-tion.[2010-12-10].http://www.w3.org/TR/200 4/REC-owl- guide-20040210/.

[8] Xiyong Zhu, Xuwei Pan, Zhengcheng Wang. Research on the Approaches to Context-based Knowledge Sharing and Reuse[J]. Journal of the China Society for Scientific and Technical Information, 2007,26(2):179-184

[9] Dwork C, Kumar R, Naor M, Sivakurmar D. Rank aggregation methods for the Web[G].Proc. of the 10th Intl. World Wide Web Conference (WWW10), Hong Kong, 2001,146-153. 